\title{
REVIEW
}

\section{Clinical review: The hospital of the future - building intelligent environments to facilitate safe and effective acute care delivery}

\author{
Brian W Pickering ${ }^{* 1,2}$, John M Litell,4, Vitaly Herasevich ${ }^{1,2}$ and Ognjen Gajic ${ }^{2,3}$
}

\begin{abstract}
The translation of knowledge into rational care is as essential and pressing a task as the development of new diagnostic or therapeutic devices, and is arguably more important. The emerging science of health care delivery has identified the central role of human factor ergonomics in the prevention of medical error, omission, and waste. Novel informatics and systems engineering strategies provide an excellent opportunity to improve the design of acute care delivery. In this article, future hospitals are envisioned as organizations built around smart environments that facilitate consistent delivery of effective, equitable, and error-free care focused on patient-centered rather than provider-centered outcomes.
\end{abstract}

\section{Introduction}

During the last century, rapid advancements in medical technology and subspecialty care have transformed acute care hospitals into highly complex institutions offering a wide variety of medical and surgical interventions. Tremendous advances in emergency and critical care medicine, surgery, and anesthesia have enabled meaningful survival among patients suffering previously lethal conditions. Unfortunately, these benefits have been to a large extent offset by iatrogenic harm and failures in care delivery due to ineffective organization. Hundreds of thousands of hospitalized patients are injured or killed each year as a result of care that is well-intended but either poorly designed or poorly implemented [1]. Inappropriate financial incentives, neglect of human factors, and the lack of a systematic approach to the science of health care delivery still pose major obstacles

*Correspondence: pickering.brian@mayo.edu

'Department of Anesthesiology, Division of Critical Care Medicine, Mayo Clinic, 200 First Street SW, Rochester, MN 55905, USA

Full list of author information is available at the end of the article to better hospital care. All too often, in our rush to advance the leading edge of science we have neglected to perfect the core interface between patients and the systems constructed to care for them.

Clearly, the mere presence of sophisticated equipment and an ever-expanding evidence base do not guarantee optimum care. These various elements must be integrated into highly organized and responsive systems that can facilitate safe, timely, consistent, and effective care. The following paragraphs discuss the barriers to safe and effective acute care delivery and highlight the changes that will shape the next generation of acute care hospitals (Figure 1).

\section{Human factors, ergonomics, and safety culture: lessons from anesthesiology}

In a landmark paper published in 1978, Cooper and colleagues [2] described using human factors research methods to retrospectively analyze almost 350 preventable anesthesia incidents. (Human factors is a multidisciplinary field seeking to understand human capabilities and to design, develop, and deploy systems and services to augment those capabilities.) Using a critical incident analysis approach, the authors determined that human factors were responsible for the vast majority of incidents with only a minority attributed to equipment failures or other factors. That preventable harm was largely attributed to human factors (at that time mostly physician related) was a radical new idea. The anesthesiology community embraced this new knowledge, using it as a foundation upon which to build a strong culture of safety and risk aversion that has had considerable impact on patient-centered outcomes. At the heart of that culture is an acknowledgment that systems of health care delivery must be designed to minimize the potential for human error. The most obvious manifestation of this change was to re-engineer the physical artifacts in the environment the anesthetic machine and physiologic monitors. But of equal importance were the re-engineering of the processes of care delivery (checklists, duty hours, rare event simulation) and mechanisms within the anesthesiology 


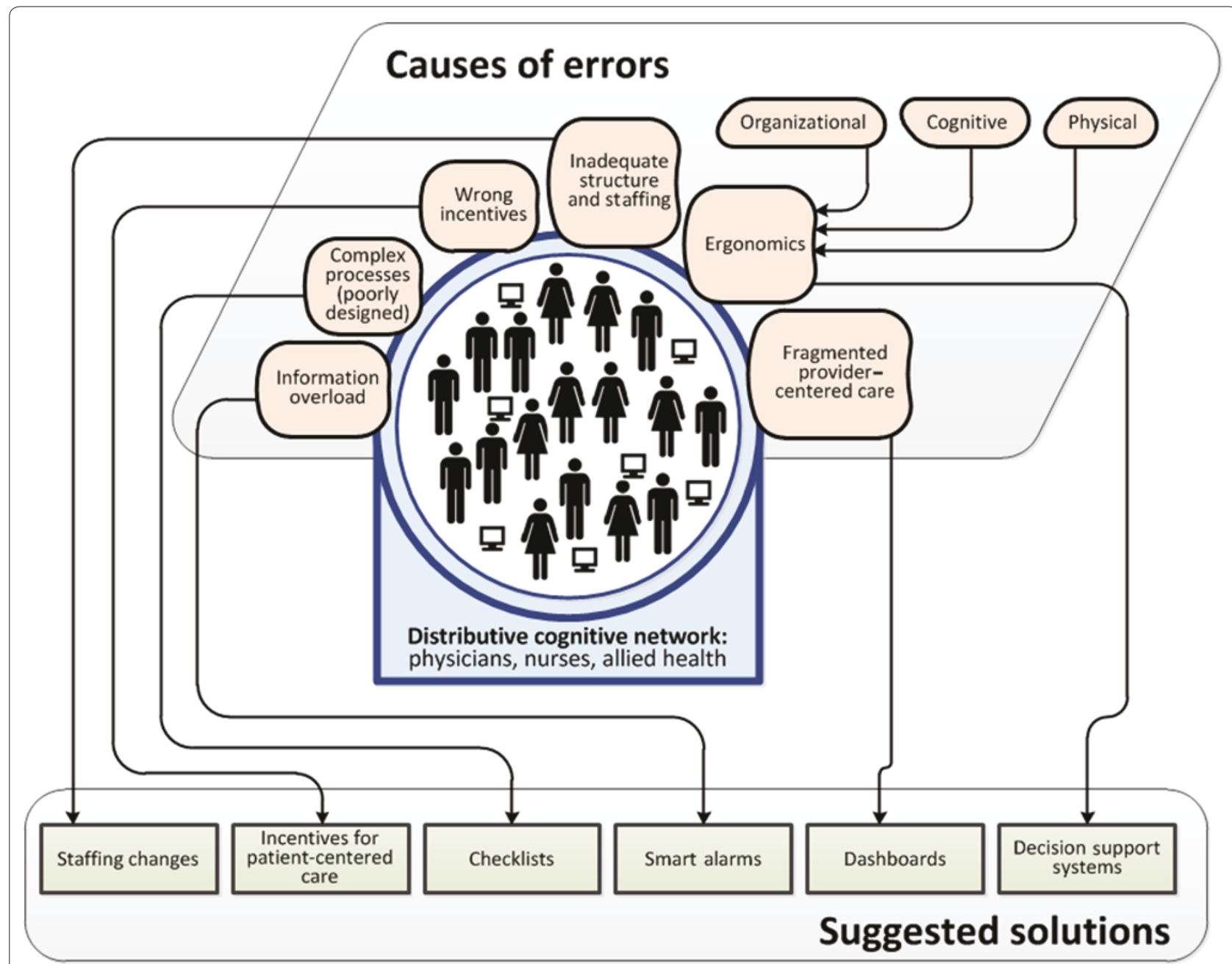

Figure 1. Challenges and opportunities for improving health care delivery in acute hospitals.

community for reporting, identifying and eliminating new errors. It would take until 1999 before the concept was more broadly articulated by the Institute of Medicine in their landmark report 'To Err is Human' [3]. What the anesthesiology response clearly highlights is the impact improved human factor ergonomics has on the ability of health care providers to perform safely within highly technical environments.

The practice of intensive care medicine shares many characteristics with anesthesiology. In terms of patient survival and resource utilization many of the most dramatic critical care interventions have derived from changes in the culture brought about by simple systemsbased tools. Prominent examples include checklists for the placement of central venous catheters, daily patient care goal lists, and clinical practice guidelines [4]. When used consistently these tools are extremely successful; however, getting providers to accept them can be very challenging [5]. The barriers to adoption of evidence-based tools are multiple but often arise from one of the three broad domains: knowledge, attitudes and behavior [6]. In 2009 the critical care community joined in the signing of the Declaration of Vienna, which expresses a commitment to improve patient outcomes through a reduction in medical error [7]. A multimodal approach incorporating human factor ergonomics will be an important component of any future strategy for eliminating errors in ICU care (Figure 1).

\section{Optimizing health care delivery with informatics and systems engineering}

Information and knowledge are the bedrock upon which we all make decisions. One change in our work environment that has the potential to facilitate communication and enforce adherence of global best practices is the transformation of the medical record from written to digital form. The role of health information technology (HIT; a technological framework for improving the 
quality, safety, and efficiency of the health care delivery system) in healthcare continues to evolve as the adoption of electronic medical records (EMRs) and information technologies becomes more widespread. Multidisciplinary teams of ICU providers make decisions and deliver care to acutely ill patients in complex environments. The interaction between providers and technology in these environments may have a profound impact on the quality of care delivered to the patient (Figure 2). The adoption of EMR has been proposed as a valuable tool for driving improvements in the quality of that care. These conclusions are based on observational and experimental studies suggesting that HIT has the potential to assist in making healthcare safer and more efficient. A recent review demonstrated that $92 \%$ of HIT studies found either a positive or mixed-positive impact on the quality of care delivered to patients [8].

It is essential to remember the nuances in these data. What is clear from the literature in this area is that HIT adoption alone is not likely to result in meaningful improvements in safety or quality. For example, while HIT offers significant advantages in terms of accessibility and legibility over paper records, this does not result directly in improved patient-centered outcomes. Even in situations where HIT would appear to offer clear advantages over paper-based alternatives, such as the availability of electronic prescribing with in-built alerts, HIT adoption has a very real potential to disrupt established practices and result in worse outcomes for patients, decreased efficiency, and increased cost $[9,10]$. In one particular study, Han and colleagues [10] reported that the adoption of a commercially available physician order entry system was associated with a two-fold increase in severity-adjusted mortality in a pediatric ICU. In order to best leverage the potential advantages of HIT adoption, a more refined understanding of how technology disrupts or supports health care providers' ability to deliver high quality care is needed. The field of systems engineering offers some promising tools that may be adapted to the study of health care delivery, providing a buffer against some of the unintended consequences of HIT adoption and highlighting new mechanisms for improving care delivery [11]. The primary task of this approach is to identify the most relevant characteristics of a system and to represent it in a mathematical model. These models can be analyzed to reveal patterns in the behavior of the system and to devise and test improvements. This process is radically different from the classical hypothesis-driven epidemiological approach to biomedical research [12]. A recently published report highlights the potential role systems engineering tools have in improving health care delivery [13].

Using sepsis treatment as an example, the systems engineer would build a model of the process of care delivery as follows: define the steps (blood cultures, fluid administration, antibiotic administration, source identification, lactate measurement, central line insertion, blood product administration, and so on); identify the system components required to deliver care (what equipment is required, who are the personnel, how is the task performed); perform a field observation study of, for example, frequency of occurrence of sepsis resuscitation, sepsis resource utilization, time taken to complete each step, process completion and failure rates. The resulting computer model would then be used to identify weaknesses, redundancies, failures, and successes in sepsis resuscitation processes and to simulate the impact of changes in those processes on outcomes such as sepsis bundle compliance rates and time to resuscitation. The advantage of this approach is that it greatly enhances our ability to identify vulnerabilities and test solutions before exposing patients to changes that may result in unanticipated harm. The future challenge for physicians and engineers is to develop a common understanding and to apply established engineering practices to the health care setting.

\section{The role of cognitive ergonomics in medical decision making}

Daily work in the ICU involves multitasking and responding to unpredictable events, such as patient decompensation and interruptions. These features combine to produce an environment in which providers must rely on implicit knowledge and internal task management schema to guide the prioritization and performance of tasks. Given what is known about human cognition and the complexity of decision making in an average ICU [14], it is objectively unreasonable to expect a human being to perform at this level without committing occasional - even regular errors. A better understanding of the interactions and behaviors that result in effective medical decision making will lead to the development and adoption of integrated decision supports that reduce the potential for diagnostic error and therapeutic harm (Figure 3).

In order to provide safe and effective critical care, the bedside provider needs to have an accurate mental model of the patient - in other words a clear picture 'in their head' of the patient's background, physiology and potential response to therapies or interventions. To develop a representative mental model, the provider must integrate a tremendous amount of disparate data elements in real time. Providers must skillfully process both 'hard' and 'soft' data sources, including tactile input during examination, interpersonal social clues from team members, visual and spatial input from clinical imaging, and algebraic reformulations of laboratory data.

Even the simplest clinical decisions may be impeded by poor information management leading to inaccurate 


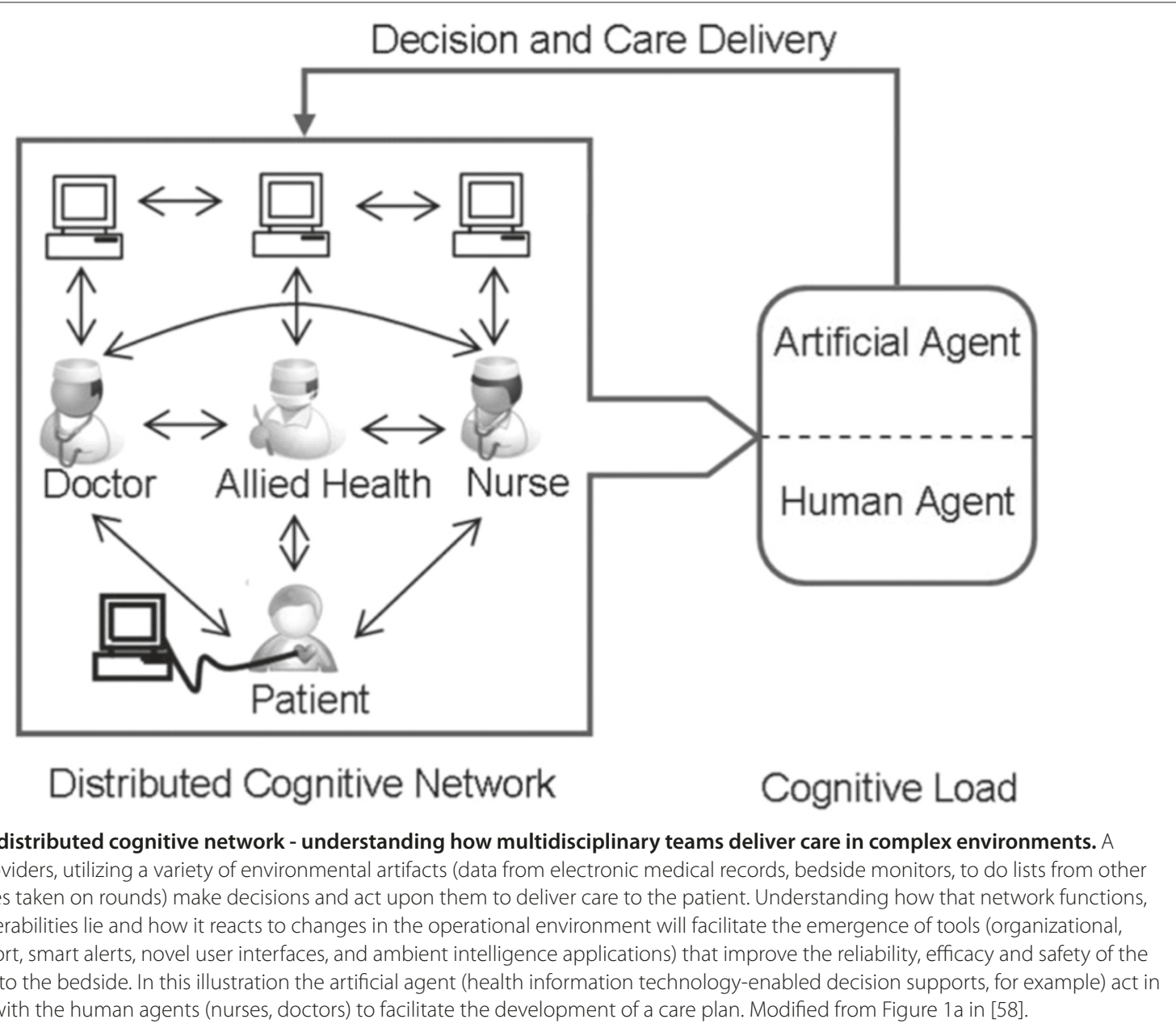

mental models. Take a very low level medical management problem such as the decision to replace electrolytes based on laboratory values: when abnormal values are differentiated from normal values by color or asterisk, for example, they are less likely to be overlooked by the provider than those that are not. In this case the cognitive ergonomic factors that influence decision making are mostly confined to how the system presents an abnormal laboratory value. (Cognitive ergonomics is an applied science concerned with the interaction between systems and human end-users, as well as the design, structure, and operation of these systems in such a way as to make them safer and more efficient.) Get that wrong and providers may fail to respond appropriately to available data. Clearly, decision making in the live ICU environment can be significantly more complex than the example above. In this case a rigorous, systematic approach is required to identify the cognitive and ergonomic factors that contribute to both good and bad decision making. Once these are identified, the decision-making environment may be optimized and team performance enhanced through the integration of relevant clinical information into the workflow.

An important additional factor to consider in the ICU setting is the multidisciplinary, collaborative nature of modern practice. Physicians, nurses, and other providers will interpret the same data sources from different vantage points. This can complicate the development of a shared mental model and impair the ongoing communication that is essential to good outcomes in critical care.

Nemeth and colleagues [15] have articulated the challenges inherent in studying the cognitive environment of health care delivery, which relies on domainspecific insights and an understanding of individual and group cognition. In seeking to deconstruct the cognitive environment of the ICU, Zhang and Norman [16] and others have invoked the theory of distributive cognition. From a systems perspective, the ICU - with its many providers, patients, physiologic monitors, diagnostic data, and family members - can be viewed as a distributed cognitive network. To understand this concept better it is 


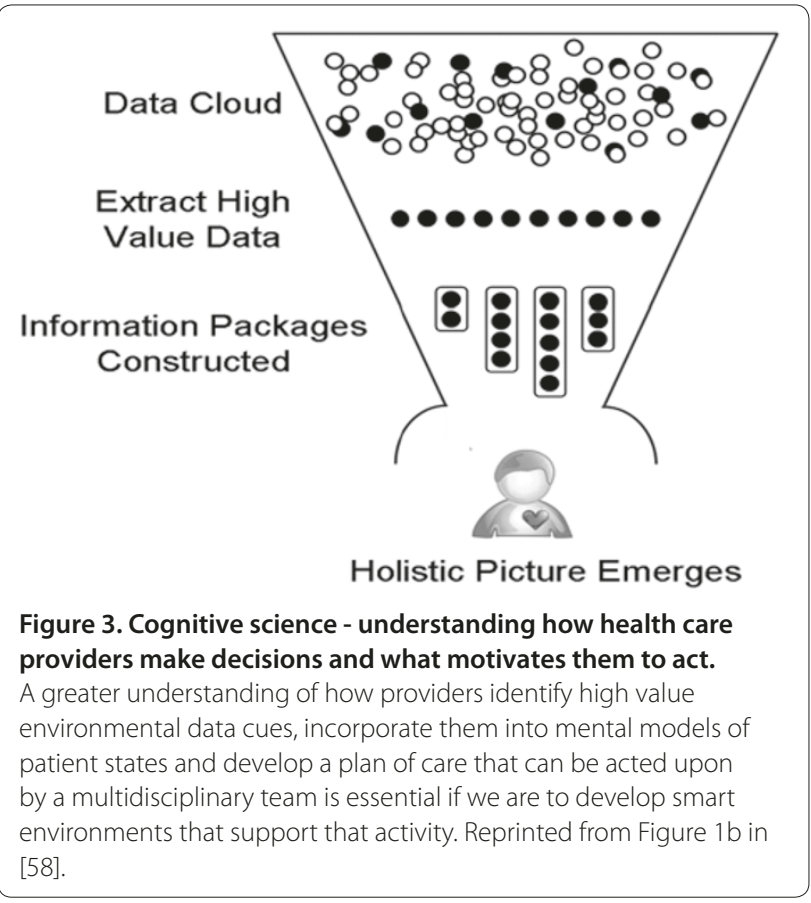

helpful to think of a multidisciplinary ICU team as it goes about the business of morning rounds (Figure 2). The individual members of the team examine the patient, look at the monitors and electronic records, talk to the family and each other and independently draw some conclusions from what they have observed. The team then come together, shares that knowledge and formulates a plan of care. That plan is then translated into actionable items that result in care being delivered to the patient.

As we move towards an increasingly digital environment, the traditional artifacts of communication (the paper chart, the end of bed flow sheet, handwritten orders, and so on) will be replaced by electronic alternatives. Depending on how those alternatives are designed and integrated into the acute care environment, HIT has the potential to disrupt or support the distributed cognitive function of the ICU team as it goes about its tasks. To operate effectively, an ICU team utilizing HIT requires both optimal functioning of providers and electronic artifacts and durable mechanisms of coordination. Individual members must understand their individual roles, the broader system, and how they fit within it. Additionally, teams need to develop shared mental models, articulate clear goals, and remain vigilant for new developments. Failure to perform in any of these areas can result in communication breakdown, error and patient harm.

Effective, open, and goal-directed communication between providers is an indispensable element of a safe and smoothly functioning critical care environment
$[17,18]$. This is reflected in the emphasis in recent years on non-technical skills - closed loop communication and role clarity - in anesthesia, emergency medicine, and critical care training modules. Expertise in communication skills is central to most high-stakes professions, and medicine is certainly no different [19]. Several authors have observed that even simple tools for standardized communication, such as rounding checklists or worksheets, have a substantial impact on the development of a shared mental model between physicians and nurses [20]. Provider satisfaction may be only one endpoint affected by these interventions. Multiple investigators evaluating the association between explicitly collaborative care models and meaningful patient outcomes have noted that mortality, length of stay, and others may benefit from a new team approach to ICU care [21].

\section{Ambient intelligence and medical error prevention}

In a review of information technology Gawande and Bates provide a vision of how it may be used to enhance patient safety [22]. The authors touch on several themes: improved communications, information access, decision support, medications safety, monitoring, and oversight. In order to realize this vision of the future - one in which high quality, complex care is safely delivered to patients we need to embrace tools and metrics with which we are only vaguely familiar. In this review we have made reference to HIT, informatics, cognitive science, ergonomics, the science of healthcare delivery, and systems engineering. These are areas of expertise with which most clinicians are only vaguely familiar. In order to leverage these important tools for safer, more effective healthcare, there is a clear need for collaboration beyond the traditional sources.

While alternative solutions exist to some of the safety and performance challenges facing the critical care community, it is the flexible and adaptive nature of an electronic infrastructure that makes it such an attractive vehicle with which to deliver point of care, contextspecific information and decision support. Ambient intelligence (Figure 4) has been described as any technology with the capacity to respond to changing contexts and to intelligently assist humans in the completion of tasks [23]. In the hospital of the future, ambient intelligence would be embedded into the work environment functioning to assist healthcare providers with safe, cost-effective delivery of high quality care to patients. With the adoption of EMRs an almost complete picture of a patient's hospitalization is available to us in a digital form. Large cohorts of such data will be used to refine and validate 'digital signatures' of provider actions, patient pre-morbid conditions, health state and response to interventions. Validated digital signatures will then be processed in data warehouses where they will be 


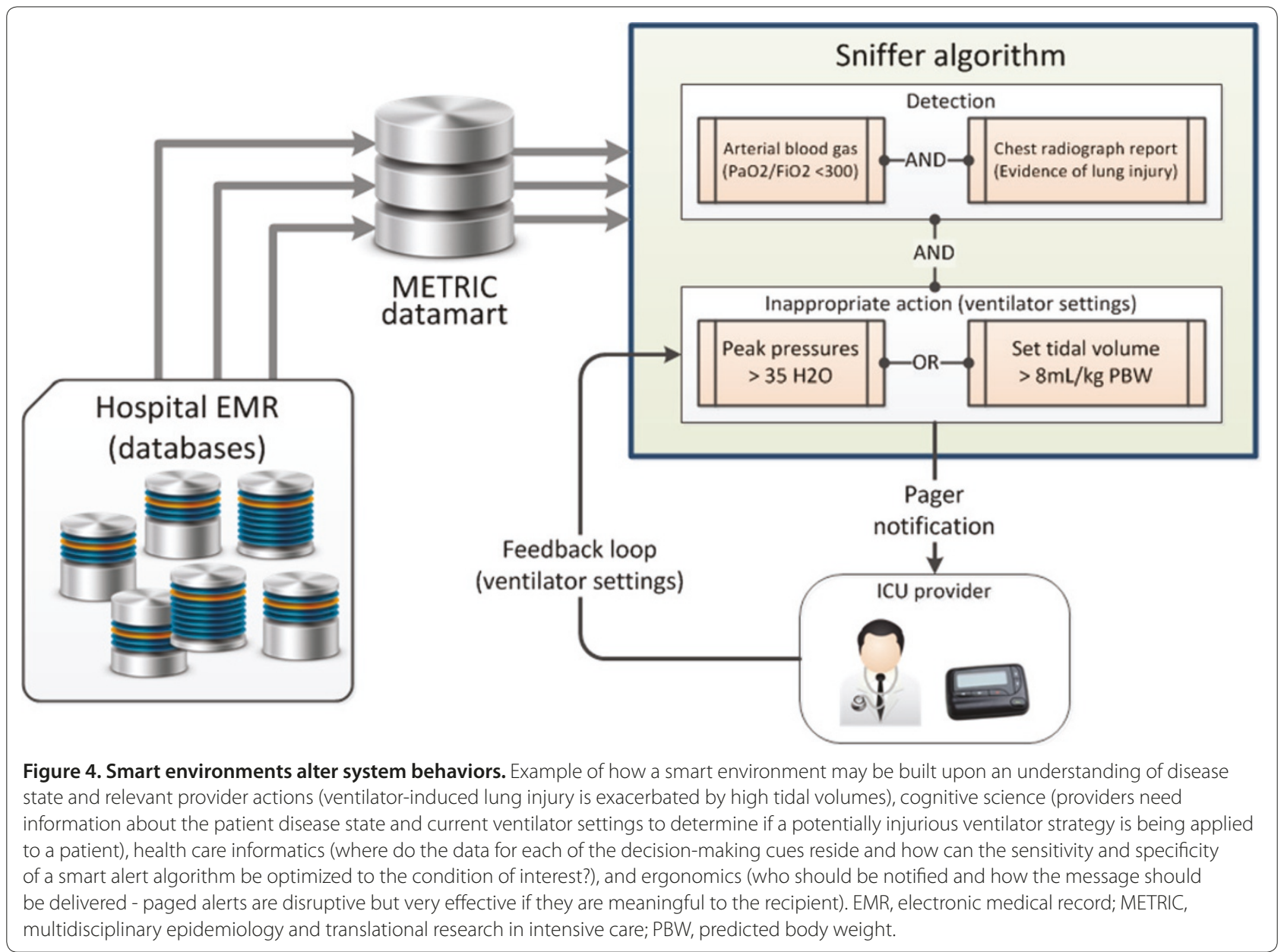

compared to expected patterns of system behavior. Bearing in mind that most errors in medicine arise from human factors rather than failures of equipment, appropriate alerts and supports will be generated when unexplained deviations from ideal behavior are detected, nudging the health care delivery system back onto an expected path.

Many of the conditions for the development of ambient intelligence ICU applications currently exist. Sources of information on patient health states and provider actions are increasingly available in their raw form through health information technologies such as comprehensive EMRs and computerized physician order entry systems. The necessary mobile technologies, allowing users to interact with information at the point of need, have been developed and tested in the form of smart phones and gesture based tablets. The key missing ingredients are valid rules that connect the available technology to digital signatures of patient health states, provider responses, and a palette of appropriate actions for the observed circumstances. The development and testing of those rules present important intellectual challenges and opportunities for health care providers building the hospitals of the future.

\section{Structural and cultural changes in the ICU of the future}

When comparing outcomes from different health systems, the question remains, 'What are the structural and cultural factors that have contributed to the reported outcomes?' Differences in outcome have been associated with factors as diverse as bed availability, 24 hour staffing models, availability of trained intensivists, closed units, engagement in clinical trials, and availability of an EMR $[24,25]$. It has even been suggested that the separation of critical care medicine from other disciplines at a national level plays an important role in determining patient outcomes [26]. What is apparent from a review of the literature is that the ideal model has yet to be defined. A scientific approach to determining best practices in critical care in the hospital of the future is required and will likely result in a shift in emphasis towards more effective care delivery models [23]. In the following we hypothesize that mechanisms for ensuring the consistent 
application of truly vital aspects of critical care - clear communication, coordinated team work, aggressive resuscitation, early mobilization, compassionate determination of goals of care, and prevention of serious complications - are likely to yield the greatest impact on meaningful outcomes.

The relationship between intensivist staffing and improved outcomes has been described in a number of studies since the 1980s $[27,28]$ but has gained renewed attention in the past decade as consumer groups have incorporated these findings into calls for quality standards in acute care hospitals. In a 1999 study investigating the impact of ICU staffing strategies on outcomes, Pronovost and colleagues [25] identified a three-fold reduction in hospital mortality among nearly 3,000 abdominal aortic aneurysm patients whose postoperative care was managed by an intensivist. The authors reported similar findings three years later in a systematic review demonstrating nearly uniform reduction in mortality and length of stay among patients receiving either mandatory intensivist consultation or closed ICU care [29]. This is supported by reports of the benefit of closed ICUs staffed by intensivists from Australian, New Zealand and European centers. One question for future hospitals is the extent to which the physician intensivist role can be supported or supplanted by highly trained nurse practitioners and physician assistants. Gershengorn and colleagues [30] recently found that such models had no detrimental impact on important safety and quality indicators. Another paradigm that will continue to be refined as we move into a digital future is that of centralized remote surveillance and decision support the telemedicine model. While on-site, 24-hour intensivist coverage might be preferred, given its impact on improved processes of care and staff satisfaction [31], whereas in some locations this coverage will need to be supplied virtually via telemedicine and tele-consultation. The culture and infrastructure of such models is still rapidly evolving, but there is accumulating evidence that ICU telemedicine may be associated with decreases in mortality and hospital length of stay, as well as improved adherence to consequential best practices and lower rates of preventable complications [32]. Several observational studies have identified the impact of additional organizational features on the quality of ICU care. Examples include annual case volume [33], the timeliness of resuscitative therapy $[34,35]$, nurse staffing and experience [36], and nurse-physician communication [37].

The implication is that unit culture, teamwork, communication, and ergonomics contribute significantly to important patient outcomes. A challenge for the future is to determine which combination of factors results in improved quality of care and outcomes among critically ill patients $[26,38]$.

\section{'Less is more' - minimizing iatrogenic waste}

A careful examination of common ICU interventions reveals a recurring theme: less is more [39]. For example, data from pulmonary artery, central venous and peripheral arterial catheters [40] are rarely necessary, frequently inaccurate, and often misinterpreted [40-43]. In future hospitals we will likely see a shift in physiologic monitoring to less invasive tools backed by alert systems that integrate closely with the patient's EMR data.

In this domain we are likely to see point-of-care ultrasonography play an increasingly central role in the bedside evaluation of critically ill patients, not only in the hands of emergency medicine physicians [44] but also of intensivists and rapid response team members. Bedside critical care ultrasound can supply actionable, real time data, with very little risk. It is essential to note, however, that an ultrasound probe wielded by an under-trained and overconfident intensivist can be dangerous in much the same way as misinterpreted pulmonary artery catheter data. As critical care ultrasound is increasingly woven into the daily practice of critical care, appropriate training will be essential to maintain patient safety.

Less is more also applies to the routine use of other time-honored diagnostic tools, such as daily chest radiographs, which often add little to the management of ICU patients (except additional cost) [45]. The less is more philosophy also has echoes in the use of noninvasive ventilation as a means to avoid endotracheal intubation in carefully selected patients. For those patients who do progress to intubation, the focus of the intensivist should be squarely on removing the tube at the earliest and safest possible moment. Discontinuation of mechanical ventilation, one of the most significant milestones for critically ill patients, is often guided by socalled 'weaning protocols.' As Hall and colleagues [46] and others have suggested, 'weaning' implies the gradual removal of a nourishing substance, and providers apply this logic to mechanical ventilation at their patient's peril. High-quality data have shown us that the best method to gauge readiness for discontinuation of mechanical ventilation is quite simply to try it $[47,48]$. Daily structured spontaneous breathing trials, with interruptions of sedation and optimization of underlying conditions, are the quickest way to assess readiness for extubation [49]. Indeed, because of associated complexity and time constraints, most other metrics of extubation readiness probably just delay extubation.

\section{Early rehabilitation and the compassionate involvement of the family}

In addition to improvements in the ergonomics and market penetration of electronic health records and decision support tools, the hospital of the future will also incorporate several low tech strategies. The care and 
rehabilitation of the critically ill will play an increasingly prominent role in future hospitals. As the population ages, financial constraints tighten, remote monitoring technology improves, and acute care needs steadily increase, inpatient space will evolve towards predominantly intensive, intermediate, and rehabilitative care.

Following the resuscitation phase of illness, patients will benefit from early physical and occupational therapy, including early mobilization in the ICU using progressively mobile portable ventilators. A randomized controlled trial by Schweickert and colleagues [50] showed that the simple act of mobilizing patients early can safely relieve the patient of two days of delirium and mechanical ventilation. Few other ICU interventions are this successful, and almost none are this cost-effective. Broadly speaking, rehabilitation medicine has made tremendous gains in delivering meaningful improvements to patients who might not otherwise transition effectively back to life outside the hospital [51]. As demand for ICU and telemetry care continues to increase, society will only benefit maximally if specialized rehabilitation services receive proportionate attention and resources.

Future hospitals will recognize the psychological stresses that afflict semi-lucid ICU patients and will make efforts to minimize them. The current epidemic of ICUacquired delirium will be increasingly recognized as a preventable syndrome with measurable effects on morbidity and mortality $[52,53]$. To address this, as well as issues of advocacy and goals of care, traditional restrictions on family visiting hours will fall away in favor of a paradigm of family inclusion [54].

The current variability in approach to end of life care discussions and decision making is the result of both social/cultural differences and lack of evidence of benefit of one approach over another. At the extremes, primarily family/patient or physician driven end of life decision making can result in either unnecessary patient suffering or a premature death. Models of collaborative decision making will need to be refined and tested for each cultural/social context. The paradigms of maximal therapy, often regardless of potential outcome or cost, or that of care withdrawal/limitation based on biased assumptions and judgments will mature to one that seeks to understand the patient and their goals in a broader psychosocial context [55]. The ideal model should strengthen opportunities to personalize and individualize care with the goal of facilitating either enhanced quality of life or a dignified transition to end of life care.

\section{Future challenges}

Before we can develop environments in which safe patient-centered care is consistently delivered, a number of challenges need to be acknowledged and, ultimately, addressed [56]. Often (no matter whether in a private insurance, single payer or mixed health care system) the broad interests of individual stake holders are in unintentional conflict with a mission of high quality patient-centered outcomes. Failure to examine and resolve the source of that conflict is the primary threat to the hospital of the future outlined in this article.

Critical care medicine remains a young specialty and as such continues to define itself along the lines of legacy founding departments such as surgery, medicine, anesthesia and nursing. The competing interests of these department-specific critical care brands can be difficult to reconcile. The impact, good and bad, of such fragmentation on patient-centered outcomes needs to be better understood before any of the changes in care delivery models outlined in this article can be implemented.

More than any new medical knowledge, incentives are the primary force that drives change. In order to move towards high quality patient-centered care, the 'right' incentives need to be put in place. How these incentives are developed and implemented is of critical importance in shaping future health care environments. The unresolved role of the government and the private sector in determining the nature of those incentives remains a critical barrier to the development of patient-centered health care systems. Conflict between stake holders can lead to perverse incentives that serve only narrow interests, while shifting the focus away from a mission of high-quality, patient-centered care.

Inherent conflict has always existed between the education and care delivery missions of hospitals. Those missions, while highly interdependent, are too often considered in isolation of each other. In the future hospital environments envisioned in this paper, patientcentered outcomes are given primacy over all else. The roles and responsibilities of learners within such a system need to be carefully evaluated and redesigned. What makes this a particularly difficult task is that the impact of system-wide changes in education models take a long time to impact on patient-centered outcomes. The impact of change is often not fully appreciated until providers, trained within the new system, come to front-line practice. If future hospitals are to continue to benefit from highly trained and motivated front-line staff, an appropriate balance between service/education and time at the bedside/lifestyle needs to be struck.

As presented in this paper, the infrastructure and resources required to support and maintain state of the art acute care environments will more often be found in well-resourced tertiary care centers than community hospitals [57]. The consequences of such changes are not known but a tiered approach to the development of critical care delivery systems will need to be developed. In this case a precedent exists in the world of trauma care 
Table 1. Knowledge deficits that must be addressed before the emergence of smart environments that facilitate reliable, safe, and compassionate delivery of appropriate interventions to hospitalized patients

\begin{tabular}{|c|c|c|}
\hline Modality & Tools & Impact \\
\hline $\begin{array}{l}\text { Human factors and } \\
\text { cognitive science }\end{array}$ & $\begin{array}{l}\text { Surveys } \\
\text { Field observation } \\
\text { Interview } \\
\text { Chart review } \\
\text { Standard ontology of error }\end{array}$ & $\begin{array}{l}\text { Identify the human factors that contribute to diagnostic, rescue, or care delivery failures } \\
\text { that result in excess morbidity and mortality in the ICU } \\
\text { Identify the modes of alert that are the most effective modifiers of behavior }\end{array}$ \\
\hline $\begin{array}{l}\text { Ergonomics and } \\
\text { engineering }\end{array}$ & $\begin{array}{l}\text { Field observation } \\
\text { Process modeling } \\
\text { Simulation }\end{array}$ & $\begin{array}{l}\text { Understand processes of care delivery } \\
\text { Identify and eliminate environmental factors that impede the delivery of care } \\
\text { Identify environmental artifacts that can be re-engineered to force best practice } \\
\text { Test the impact of changes in models of care delivery } \\
\text { Test the impact on processes of care in high-fidelity simulation environments }\end{array}$ \\
\hline $\begin{array}{l}\text { Health care informatics } \\
\text { and health information } \\
\text { technology }\end{array}$ & $\begin{array}{l}\text { Data warehousing } \\
\text { Epidemiology } \\
\text { Data mining } \\
\text { Social networks }\end{array}$ & $\begin{array}{l}\text { Reliably capture digital signatures of patient conditions and provider actions } \\
\text { Build real-time feedback to systems of health care delivery (provider and manager) } \\
\text { Facilitate the reporting of errors } \\
\text { Facilitate secondary data use (for example, the analysis of large data sets from multiple } \\
\text { care delivery settings) } \\
\text { Dissemination of knowledge }\end{array}$ \\
\hline Culture & $\begin{array}{l}\text { Reporting error at a local level } \\
\text { Coordinated response of ICU } \\
\text { community to error } \\
\text { Lobbying } \\
\text { Funding } \\
\text { Developing and enforcing standards }\end{array}$ & $\begin{array}{l}\text { Knowledge of new or unanticipated errors } \\
\text { Organized response re-enforces value of reporting } \\
\text { Facilitate the implementation of recommendations } \\
\text { Reform of incentives } \\
\text { Perpetuate the safety culture }\end{array}$ \\
\hline
\end{tabular}

and lessons learned during the development of that model should be adapted to critical care.

Maintaining the continuity of clinical care and integrity of patient information as they transition from one health care setting to the next represents an enormous challenge for hospital practitioners of the future. Once the technical and privacy issues are resolved it is likely that the increased availability of EMR systems will improve access to data from other care areas and that the data will increasingly be viewed as belonging to the patient rather than the health care provider. In addition, the development of non-invasive monitors and ICU outreach services will reduce the likelihood that developing physiologic instability will be overlooked. With more data being generated from patients in general care wards it is likely that surveillance algorithms or data 'sniffers' will be utilized to issue alerts to bedside providers in the event of uncorrected deviations from the expected clinical course.

Finally, while it is true that the management of increasing amounts of clinical data and knowledge will be best supported by well designed health information systems, we must not forget the central role the front line provider will continue to play as the gatekeeper to decision making and care delivery for the foreseeable future. The bedside provider accesses an array of decisionmaking cues that are not well represented in the current generation of electronic medical records. Their compassion, intuition and experience are brought to bear on that information and they draw conclusions that currently remain beyond the capability of the most sophisticated decision support tools. What will change is how digital
Table 2. Emerging concepts that will define next-generation acute care hospitals

Safety culture

Ambient intelligence

Human factor engineering and ergonomics

'Less is more' - eliminate iatrogenic waste

Noninvasive monitoring/testing (bedside ultrasound)

Staffing/regionalization/telemedicine/physician extenders

Patient/family-centered care

Inpatient services primarily ICU, intermediate, and rehabilitation

information is accessed. It is no longer reasonable to expect a provider to search through a mass of data to find the relevant information. Increasingly that data will be pre-processed and presented in task-specific data views that suppress the 'noise' in favor of the relevant 'signal.'

\section{Conclusion}

Both the integrity of the healing professions and the safety of our patients hinge on our ability to identify and overcome barriers to effective, error-free care delivery. These barriers are not due to the lack of a sophisticated diagnostic and therapeutic armamentarium. Rather, the true challenge faced when designing hospitals of the future lies in studying and improving the manner in which we utilize and implement medical knowledge and technology (Table 1). In this article we have reviewed emerging concepts from the fields of health care delivery science, ergonomics, cognitive science, informatics, and 
system engineering (Table 2). We have suggested how these concepts may be utilized to develop next generation acute care environments. The key characteristic of these environments will be the support of patient-centered rather than provider-centered care delivery. In other words, the provision of safe, effective, error-free, and compassionate care to acutely ill or injured patients. Complexity, human factors, cultural barriers, and the inertia inherent in established structures and processes will make the task of constructing that environment far from easy. However, we have no doubt that change is coming and will gain momentum quickly once the first steps are taken.

\section{Abbreviations}

EMR, electronic medical record; HIT, health information technology.

\section{Acknowledgments}

We sincerely thank Dr Rolf D Hubmayr for reviewing the manuscript and providing helpful suggestions and insights.

\section{Author details}

'Department of Anesthesiology, Division of Critical Care Medicine, Mayo Clinic, 200 First Street SW, Rochester, MN 55905, USA. ${ }^{2}$ Multidisciplinary Epidemiology and Translational Research in Intensive Care, Mayo Clinic, 200 First Street SW, Rochester, MN 55905, USA. ${ }^{3}$ Department of Pulmonology and Critical Care Medicine, Mayo Clinic, 200 First Street SW, Rochester, MN 55905, USA. ${ }^{4}$ Critical Care Medicine, Mayo Clinic, 200 First Street SW, Rochester, MN 55905, USA.

Published: 26 April 2012

\section{References}

1. Kohn K, Corrigan J, Donaldson M: To Err is Human: Building a Safer Health System. Chapter 2. Washington, DC: National Academy Press; 1999: 26-48.

2. Cooper JB, Newbower RS, Long CD, McPeek B: Preventable anesthesia mishaps: a study of human factors. Anesthesiology 1978, 49:399-406.

3. Kohn L, Corrigan, JM, Donaldson MS: To Err Is Human: Building a Safer Health System. Washington, DC: National Academy Press; 1999.

4. Haynes AB, Weiser TG, Berry WR, Lipsitz SR, Breizat AH, Dellinger EP, Herbosa T, Joseph S, Kibatala PL, Lapitan MC, Merry AF, Moorthy K, Reznick RK, Taylor B, Gawande AA; Safe Surgery Saves Lives Study Group: A surgical safety checklist to reduce morbidity and mortality in a global population. N Engl J Med 2009, 360:491-499.

5. Levy MM, Pronovost PJ, Dellinger RP, Townsend S, Resar RK, Clemmer TP, Ramsay G: Sepsis change bundles: converting guidelines into meaningful change in behavior and clinical outcome. Crit Care Med 2004, 32(11 Suppl):S595-597.

6. Cabana MD, Rand CS, Powe NR, Wu AW, Wilson MH, Abboud PA, Rubin HR: Why don't physicians follow clinical practice guidelines? A framework for improvement. JAMA 1999, 282:1458-1465.

7. Moreno RP, Rhodes A, Donchin Y: Patient safety in intensive care medicine: the Declaration of Vienna. Intensive Care Med 2009, 35:1667-1672.

8. Buntin MB, Burke MF, Hoaglin MC, Blumenthal D: The benefits of health information technology: a review of the recent literature shows predominantly positive results. Health Aff (Millwood) 2011, 30:464-471.

9. Ash JS, Berg M, Coiera E: Some unintended consequences of information technology in health care: the nature of patient care information systemrelated errors. J Am Med Inform Assoc 2004, 11:104-112.

10. Han YY, Carcillo JA, Venkataraman ST, Clark RS, Watson RS, Nguyen TC, Bayir H, Orr RA: Unexpected increased mortality after implementation of a commercially sold computerized physician order entry system. Pediatrics 2005, 116:1506-1512.

11. Sittig DF, Ash JS, Zhang J, Osheroff JA, Shabot MM: Lessons from "Unexpected increased mortality after implementation of a commercially sold computerized physician order entry system". Pediatrics 2006, 118:797-801.
12. Kopach-Konrad R, Lawley M, Criswell M, Hasan I, Chakraborty S, Pekny J, Doebbeling BN: Applying systems engineering principles in improving health care delivery. J General Int Med 2007, 22 Suppl 3:431-437.

13. Proctor P, Compton W, Crossman J, Fanjiang G: Building a Better Delivery System: a New Engineering/Health Care Partnership, Committee on Engineering and the Health Care System. Washington DC: National Academy of Engineering and Institute of Medicine, National Academy Press; 2005.

14. Patel VL, Zhang J, Yoskowitz NA, Green R, Sayan OR: Translational cognition for decision support in critical care environments: a review. J Biomed Inform 2008, 41:413-431.

15. Nemeth C, O'Connor M, Klock P, Cook R: Discovering healthcare cognition: The use of cognitive artifacts to reveal cognitive work. Organization Studies 2006, 27:1011-1035.

16. Zhang J, Norman D: Representations in distributed cognitive tasks. Cogn Sci 1994, 18:87-122.

17. Sutcliffe KM, Lewton E, Rosenthal MM: Communication failures: an insidious contributor to medical mishaps. Acad Med 2004, 79:186-194.

18. Alvarez G, Coiera E: Interdisciplinary communication: an uncharted source of medical error? J Crit Care 2006, 21:236-242; discussion 242.

19. Brindley PG, Reynolds SF: Improving verbal communication in critical care medicine. J Crit Care 2011, 26:155-159.

20. Pickering BW, Hurley K, Marsh B: Identification of patient information corruption in the intensive care unit: using a scoring tool to direct quality improvements in handover. Crit Care Med 2009, 37:2905-2912.

21. Narasimhan M, Eisen LA, Mahoney CD, Acerra FL, Rosen MJ: Improving nurse-physician communication and satisfaction in the intensive care unit with a daily goals worksheet. Am J Crit Care 2006, 15:217-222.

22. Bates DW, Gawande AA: Improving safety with information technology. N Engl J Med 2003, 348:2526-2534.

23. Pronovost PJ, Goeschel CA: Viewing health care delivery as science: challenges, benefits, and policy implications. Health Serv Res 2010, 45:1508-1522.

24. Pronovost PJ, Holzmueller CG, Clattenburg L, Berenholtz S, Martinez EA, Paz JR, Needham DM: Team care: beyond open and closed intensive care units. Curr Opin Crit Care 2006, 12:604-608.

25. Pronovost PJ, Jenckes MW, Dorman T, Garrett E, Breslow MJ, Rosenfeld BA, Lipsett PA, Bass E: Organizational characteristics of intensive care units related to outcomes of abdominal aortic surgery. JAMA 1999, 281:1310-1317.

26. Bellomo R, Stow PJ, Hart GK: Why is there such a difference in outcome between Australian intensive care units and others? Curr Opin Anaesthesiol 2007, 20:100-105.

27. Reynolds HN, Haupt MT, Thill-Baharozian MC, Carlson RW: Impact of critical care physician staffing on patients with septic shock in a university hospital medical intensive care unit. JAMA 1988, 260:3446-3450.

28. Gajic O, Afessa B: Physician staffing models and patient safety in the ICU. Chest 2009, 135:1038-1044.

29. Pronovost PJ, Angus DC, Dorman T, Robinson KA, Dremsizov TT, Young TL: Physician staffing patterns and clinical outcomes in critically ill patients: a systematic review. JAMA 2002, 288:2151-2162.

30. Gershengorn HB, Wunsch H, Wahab R, Leaf D, Brodie D, Li G, Factor P: Impact of nonphysician staffing on outcomes in a medical ICU. Chest 2011, 139:1347-1353.

31. Gajic O, Afessa B, Hanson AC, Krpata T, Yilmaz M, Mohamed SF, Rabatin JT, Evenson LK, Aksamit TR, Peters SG, Hubmayr RD, Wylam ME: Effect of 24-hour mandatory versus on-demand critical care specialist presence on quality of care and family and provider satisfaction in the intensive care unit of a teaching hospital. Crit Care Med 2008, 36:36-44

32. Lilly CM, Cody S, Zhao H, Landry K, Baker SP, Mcllwaine J, Chandler MW, Irwin RS: Hospital mortality, length of stay, and preventable complications among critically ill patients before and after tele-ICU reengineering of critical care processes. JAMA 2011, 305:2175-2183.

33. Peelen $L$, de Keizer NF, Peek N, Scheffer GJ, van der Voort PH, de Jonge E: The influence of volume and intensive care unit organization on hospital mortality in patients admitted with severe sepsis: a retrospective multicentre cohort study. Crit Care 2007, 11:R40.

34. Jones AE, Brown MD, Trzeciak S, Shapiro NI, Garrett JS, Heffner AC, Kline JA: The effect of a quantitative resuscitation strategy on mortality in patients with sepsis: a meta-analysis. Crit Care Med 2008, 36:2734-2739.

35. Kumar A, Roberts D, Wood KE, Light B, Parrillo JE, Sharma S, Suppes R, Feinstein D, Zanotti S, Taiberg L, Gurka D, Kumar A, Cheang M: Duration of 
hypotension before initiation of effective antimicrobial therapy is the critical determinant of survival in human septic shock. Crit Care Med 2006, 34:1589-1596

36. Dennison CR, Mendez-Tellez PA, Wang W, Pronovost PJ, Needham DM: Barriers to low tidal volume ventilation in acute respiratory distress syndrome: survey development, validation, and results. Crit Care Med 2007, 35:2747-2754.

37. Baggs JG, Schmitt MH, Mushlin Al, Mitchell PH, Eldredge DH, Oakes D, Hutson AD: Association between nurse-physician collaboration and patient outcomes in three intensive care units. Crit Care Med 1999, 27:1991-1998

38. Kahn JM: Volume, outcome, and the organization of intensive care. Crit Care 2007, 11:129.

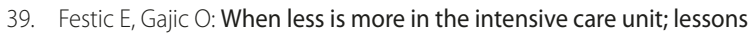
learned. Bosnian J Basic Med Sc 2009, 9 Suppl 1:54-58.

40. Garland A, Connors AF Jr: Indwelling arterial catheters in the intensive care unit: necessary and beneficial, or a harmful crutch? Am J Respir Crit Care Med 2010, 182:133-134.

41. Marik PE, Baram M, Vahid B: Does central venous pressure predict fluid responsiveness? A systematic review of the literature and the tale of seven mares. Chest 2008, 134:172-178.

42. Forrester JS, Diamond G, McHugh TJ, Swan HJ: Filling pressures in the right and left sides of the heart in acute myocardial infarction. A reappraisal of central-venous-pressure monitoring. N Engl J Med 1971, 285:190-193.

43. Shah MR, Hasselblad V, Stevenson LW, Binanay C, O'Connor CM, Sopko G, Califf RM: Impact of the pulmonary artery catheter in critically ill patients: meta-analysis of randomized clinical trials. JAMA 2005, 294:1664-1670

44. Kendall JL, Hoffenberg SR, Smith RS: History of emergency and critical care ultrasound: the evolution of a new imaging paradigm. Crit Care Med 2007, 35(5 Suppl):S126-130

45. Oba Y, Zaza T: Abandoning daily routine chest radiography in the intensive care unit: meta-analysis. Radiology 2010, 255:386-395.

46. Hall JB, Schmidt GA, Wood LDH: Principles of Critical Care. 3rd edition. New York: McGraw-Hill, Medical Pub. Division; 2005.

47. Esteban A, Alía I, Tobin MJ, Gil A, Gordo F, Vallverdú I, Blanch L, Bonet A, Vázquez A, de Pablo R, Torres A, de La Cal MA, Macías S: Effect of spontaneous breathing trial duration on outcome of attempts to discontinue mechanical ventilation. Spanish Lung Failure Collaborative Group. Am J Respir Crit Care Med 1999, 159:512-518.

48. Yang KL, Tobin MJ: A prospective study of indexes predicting the outcome of trials of weaning from mechanical ventilation. N Eng/J Med 1991, 324:1445-1450.
49. Hess DR, Maclntyre NR: Ventilator discontinuation: why are we still weaning? Am J Respir Crit Care Med 2011, 184:392-394.

50. Schweickert WD, Pohlman MC, Pohlman AS, Nigos C, Pawlik AJ, Esbrook CL, Spears L, Miller M, Franczyk M, Deprizio D, Schmidt GA, Bowman A, Barr R, McCallister KE, Hall JB, Kress JP: Early physical and occupational therapy in mechanically ventilated, critically ill patients: a randomised controlled trial. Lancet 2009, 373:1874-1882.

51. Wade DT, de Jong BA: Recent advances in rehabilitation. BMJ 2000 , 320:1385-1388.

52. Ely EW, Inouye SK, Bernard GR, Gordon S, Francis J, May L, Truman B, Speroff T, Gautam S, Margolin R, Hart RP, Dittus R: Delirium in mechanically ventilated patients: validity and reliability of the confusion assessment method for the intensive care unit (CAM-ICU). JAMA 2001, 286:2703-2710.

53. Ely EW, Shintani A, Truman B, Speroff T, Gordon SM, Harrell FE, Jr., Inouye SK, Bernard GR, Dittus RS: Delirium as a predictor of mortality in mechanically ventilated patients in the intensive care unit. JAMA 2004, 291:1753-1762.

54. Berwick DM, Kotagal M: Restricted visiting hours in ICUs: time to change. JAMA 2004, 292:736-737.

55. Muni S, Engelberg RA, Treece PD, Dotolo D, Curtis JR: The influence of race/ ethnicity and socioeconomic status on end-of-life care in the ICU. Chest 2011, 139:1025-1033.

56. Barnato AE, Kahn JM, Rubenfeld GD, McCauley K, Fontaine D, Frassica JJ, Hubmayr R, Jacobi J, Brower RG, Chalfin D, Sibbald W, Asch DA, Kelley M, Angus DC: Prioritizing the organization and management of intensive care services in the United States: the PrOMIS Conference. Crit Care Med 2007, 35:1003-1011.

57. Peek GJ, Clemens F, Elbourne D, Firmin R, Hardy P, Hibbert C, Killer H, Mugford $M$, Thalanany M, Tiruvoipati R, Truesdale A, Wilson A: CESAR: conventional ventilatory support vs extracorporeal membrane oxygenation for severe adult respiratory failure. BMC Health Serv Res 2006, 6:163.

58. Pickering BW, Litell JM, Gajic O: Ambient intelligence in the intensive care unit: designing the electronic medical record of the future. In Annual Update in Intensive Care and Emergency Medicine 2011. Edited by Vincent J-L. Springer; 2011:793-802

doi:10.1186/cc11142

Cite this article as: Pickering BW, et al: Clinical review: The hospital of the future - building intelligent environments to facilitate safe and effective acute care delivery. Critical Care 2012, 16:220. 